\title{
Evaluating Performance Benefits of Head Tracking in Modern Video Games
}

\author{
Arun Kulshreshth \\ Department of EECS \\ University of Central Florida \\ 4000 Central Florida Blvd \\ Orlando, FL 32816, USA \\ arunkul@knights.ucf.edu
}

\author{
Joseph J. LaViola Jr. \\ Department of EECS \\ University of Central Florida \\ 4000 Central Florida Blvd \\ Orlando, FL 32816, USA \\ jjl@eecs.ucf.edu
}

\begin{abstract}
We present a study that investigates user performance benefits of using head tracking in modern video games. We explored four different carefully chosen commercial games with tasks which can potentially benefit from head tracking. For each game, quantitative and qualitative measures were taken to determine if users performed better and learned faster in the experimental group (with head tracking) than in the control group (without head tracking). A game expertise pre-questionnaire was used to classify participants into casual and expert categories to analyze a possible impact on performance differences. Our results indicate that head tracking provided a significant performance benefit for experts in two of the games tested. In addition, our results indicate that head tracking is more enjoyable for slow paced video games and it potentially hurts performance in fast paced modern video games. Reasoning behind our results is discussed and is the basis for our recommendations to game developers who want to make use of head tracking to enhance game experiences.
\end{abstract}

\section{Categories and Subject Descriptors}

H.5.2 [User Interfaces]: Evaluation/methodology; K.8.0 [Personal Computing]: Games

\section{Keywords}

Head Tracking; Motion control; video games; TrackIR 5; 3D interaction; user performance \& experience.

\section{General Terms}

Experimentation, Measurement, Performance

\section{INTRODUCTION}

With the advancement of game interface technology, several new devices and gaming platforms (e.g., Microsoft Kinect,

Permission to make digital or hard copies of all or part of this work for personal or classroom use is granted without fee provided that copies are not made or distributed for profit or commercial advantage and that copies bear this notice and the full citation on the first page. To copy otherwise, to republish, to post on servers or to redistribute to lists, requires prior specific permission and/or a fee.

SUI '13, July 20-21, 2013, Los Angeles, California, USA

Copyright 2013 ACM 978-1-4503-2141-9/13/07 ...\$10.00.
PlayStation Move, TrackIR 5) that support 3D spatial interaction have been implemented and made available to consumers. Head tracking is one example of an interaction technique, commonly used in the virtual and augmented reality communities $[2,7,9]$, that has potential to be a useful approach for controlling certain gaming tasks. Recent work on head tracking and video games has shown some potential for this type of gaming interface. For example, Sko et al. [10] proposed a taxonomy of head gestures for first person shooter (FPS) games and showed that some of their techniques (peering, zooming, iron-sighting and spinning) are useful in games. In addition, previous studies [13, 14] have shown that users experience a greater sense of "presence" and satisfaction when head tracking is present. However, these studies were conducted in simple game scenarios. We seek to systematically explore the effects of head tracking, in complex gaming environments typically found in commercial video games, in order to find if there are any performance benefits and how it affects the user experience. A thorough understanding of the possible performance benefits and reasoning behind them would help game developers to make head tracked games not only more enjoyable, but more effective. Our study is an initial step towards a foundational understanding of the potential performance benefits of head tracking in modern video games.

In this paper, we present a study investigating whether user performance is enhanced when head tracking is used over a traditional button-based controller in modern video games. We made use of the TrackIR 5 head tracking device, a PC, the Xbox 360 controller for Windows, and four carefully chosen games as a representation of modern head tracking enabled games. All these games had native support for the TrackIR 5 and had tasks that may potentially benefit from use of a head tracking device. To evaluate the players performance, we collected both quantitative data based on the tasks associated with each game and qualitative data based on post-questionnaires to evaluate perception of their performance. We used a between subjects design where the control group played the games without head tracking and the experimental group played with head tracking with both groups using the Xbox 360 controller as the input device.

\section{RELATED WORK}

Sko et al. [10] used head tracking for FPS games and presented a simple two-level taxonomy, which categorized head controlled based techniques into ambient or control. Am- 
bient (or perceptual) techniques enhance the visual and/or audio feedback based on the user's head position, and control techniques are focused on the controlling the state of the game. Four interaction techniques (zooming, spinning, peering, and iron-sighting) were developed for control and two (head-coupled perspective and handy-cam) for ambient interactions. Their evaluation found that control based techniques are most useful for games which are specifically designed with head tracking in mind and ambient techniques bring more energy and realism in FPS games. However, the main focus of their work was to analyze the effectiveness of each individual technique in isolation and no quantitative measures were involved. In our study we focused on quantitatively measuring the combined affect, on user performance, of simultaneously using several techniques. Yim et al. [14] developed a low cost head tracking solution based upon the popular work of Johnny Lee [6] using Nintendo Wii Remotes. Although they did not perform a formal user study, their preliminary results show that users perceived head tracking as a more enjoyable and intuitive gaming experience.

Head gesture recognition techniques based on face tracking, which is similar to head tracking, have been studied by HCI researchers as an input to computer games. Wang et al. [13] used face tracking for head gesture recognition and developed two basic interaction techniques in two game contexts (avatar appearance \& control in a third person game and dodging-and-peeking in a FPS game). Their evaluation, based on simple game prototypes they developed, showed that the test participants experienced a greater sense of presence and satisfaction with their head tracking technique. However, they did not find any differences in user performance compared to using a traditional game controller. Limited accuracy of the head tracking data based on web cam could have been the reason that they did not find any quantifiable performance benefits.

Ashdown et al. [1] explored head tracking to switch the mouse pointer between monitors in a multi-monitor environment. Although participants preferred using head tracking, their results indicate that the task time was increased with head tracking usage. Another study [11] evaluated exaggerated head-coupled camera motions for game-like object movement but did not find any performance differences with different exaggeration levels. Zhu et al. [15] used head tracking for remote camera control but did not find any benefits of using head tracking compared to keyboard based control. Additionally, they found that users with more gaming experience performed better not only in keyboard controls but also in head tracking controls.

Head tracking has been explored by virtual reality scientists to visualize and understand complex $3 \mathrm{D}$ structures [9]. Bajura et al.[2] used head tracking for visualizing patient ultrasound data overlapped with a patient image in real time using a head mounted display (HMD). Head tracking has also been used to control avatars in Virtual Environments (VE) [7] and it was found that although head tracking is more intuitive for view control, it does not provide any performance benefits compared to using traditional button based controllers.

None of the work mentioned above evaluated performance benefits of head tracking in complex gaming environments like in modern video games. To the best of our knowledge, our work is the first to systematically explore user performance benefits of head tracking in commercially available modern video games for different game genres.

\section{SELECTING THE GAMES}

We chose the TrackIR 5 by NaturalPoint Inc. as our head tracking device because it is natively supported in many (about 130) commercially available games (a list of commercially supported games is available on the TrackIR website [8]). TrackIR 5 is an optical motion tracking game controller which can track head motions up to six degrees of freedom, but not all degrees of freedom are supported in all games, depending on the nature of interaction required for that game. Most of these games fall into three categories, racing, flight simulation, and first person shooter. We rejected the games which used head tracking for minimal tasks not related to the objective of the game. We also rejected some old games which did not support rendering at full 1080p resolution. We chose four games, Arma II, Dirt 2, Microsoft Flight and Wings of Prey, that we thought could benefit when played in head tracked environment (see Figure 1). All these games supported alternate control methods, using joystick or buttons on Xbox 360 controller, when head tracking is not available.

Arma II is a first person shooter (FPS) in which users can rotate their heads to look around in the game environment and move their heads closer to screen, in iron-sight (aim using markers on the gun) mode, to shoot distant enemies. We felt that knowledge of the ambient environment, through the use of natural gestures to look around, might help user to find enemies more easily, and zoom-in by moving closer to the screen would make the game more immersive.

Dirt $\mathbf{2}$ is a car racing game and supports head tracking only in first person view. In this game, users can rotate their heads to rotate the driver's head in the game to look around through the car windows. We expected that this would help users to see upcoming turns more easily and increase their gaming performance.

Microsoft Flight is a flight simulation game and supports head tracking in cockpit view (first person view) mode. In this game, users can also rotate their heads to look around through the windows of the cockpit. Use of head tracking would make it easier for the user to look around for any stationary objects in the flight path in order to avoid collisions.

Wings of Prey is an air combat game in which users shoot enemies while flying. This game is significantly different from Microsoft Flight because in this game you have to shoot moving targets requiring more head usage to find those targets around you. In this game, users can look around through the aircraft windows by rotating their head. The aircraft had windows to the left, right, front and top of the player. Looking around naturally would help users find surrounding enemies in the air more easily and would help them increase their performance.

\section{USER STUDY}

We conducted an experiment with four PC games (as discussed in the previous section) where participants played each game either with head tracking or without head tracking using the Xbox 360 controller. We examined both quantitative metrics, based on each game's goals and tasks, and 
qualitative metrics, based on whether participants preferred playing the games with head tracking and whether they perceived any benefits. Based on previous findings in related work and our analysis of the games, we have following hypotheses:

Hypothesis 1 (H1) : Head tracking improves user's gaming performance compared to a traditional game controller.

Hypothesis 2 (H2) : Users will learn to play games faster with head tracking on average than with a traditional game controller.

Hypothesis 3 (H3) : Users prefer playing games with head tracking since it provides a more engaging user experience.

\subsection{Participants and Equipment}

Forty participants (36 males and 4 females ranging in age from 18 to 30 with a mean age of 20.9) were recruited from a university population. A modified version of Terlecki and Newcombe's Video Game Experience survey [12] was used as a pre-questionnaire in which they answered questions about their previous gaming experience. The survey was modified to include questions related to previous experience, if any, with head tracking, and the games used for the study. Of the 40 participants, 6 were ranked as beginners ( 4 in head tracked group and 2 in non-head tracked group), 16 as intermediate ( 7 in head tracked group and 9 in non-head tracked group), and 18 as advanced (9 in each group). Since there were only a few beginners, we decided to combine beginners and intermediate categories into one category called casual gamers. The experiment duration ranged from 60 to 80 minutes depending on how long participants took to complete the tasks presented to them in the games and how much time was spent on the questionnaires. All participants were paid $\$ 10$ for their time.

The head tracked setup (see Figure 1) used a TrackIR 5 with Pro Clip, a Samsung 50" DLP 3D HDTV, a Xbox 360 controller, and a PC (Core i7 920 CPU, GTX 470 graphics card, 16 GB RAM). These are all commodity hardware components.. For the control group, the TrackIR 5 was not used and the participant played only using the Xbox 360 controller. Note that a limitation with head tracking based game camera control is that the maximum amount of head rotation is dependent on the display screen size and distance of user from screen. Too much head rotation could lead you to look away from the screen. This is the reasoning behind our use of a large screen TV for our experiments so, even if users (sitting approximately 3 feets away from the TV screen) rotate their head slightly (about 45 degree in either direction), they would still be looking at the screen.

\subsection{Experimental Task}

The participants were given the task of playing through levels of the four games. For each game, they were presented with a task specific to that game and a goal for completing each task. Participants played these games in random order (counter-balanced Latin Squares design) with three attempts for each game.

Arma II: Participants played "Single player scenario: Trial by Fire" and their task was to shoot as many enemies as possible within 10 minutes. The trial ends before 10 minutes if the player gets shot by the enemy. The game was reset after each trial.

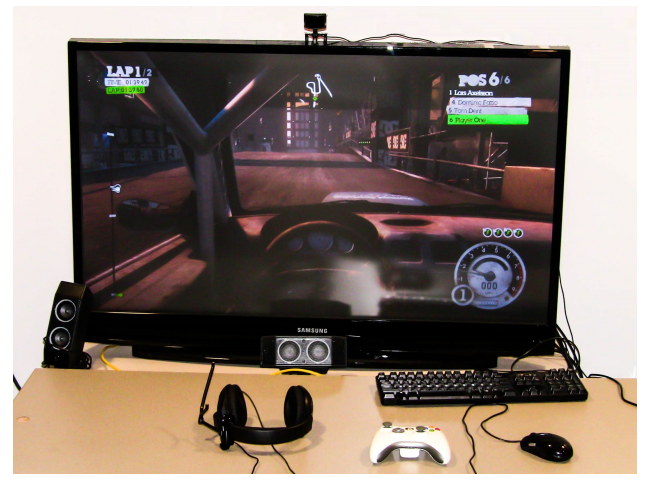

Figure 1 The experimental setup.

Table 1 Summary of metrics for each game. The metrics are used to quantify how users in the head tracked (H) and non-head tracked (NH) groups performed.

\begin{tabular}{|l|l|}
\hline \multicolumn{1}{|c|}{ Game } & Metric \\
\hline Arma II & Number of enemies shot, Survival Time \\
\hline Dirt2 & Race completion time, Rank in the race \\
\hline Microsoft Flight & Game Score \\
\hline Wings of Prey & Time taken, Number of enemy planes shot \\
\hline
\end{tabular}

Dirt 2: The participants played "London Rally" and their task was to win the race in as little time as possible with a maximum of 10 minutes. The game was reset after each trial.

Microsoft Flight: Participants played "First Flight" and their task was to maneuver the aircraft through numerous stationary balloons and finally land on the runway. The aircraft crashes if hit by balloon or if the orientation/speed of aircraft is not right while landing. The game was reset after each trial.

Wings of Prey: The participants played single player mission "Battle of Britain: Defend Manston" and their task was to shoot down all the enemy planes before time runs out (about 5 minutes). The game ends before the time limit if the aircraft crashes or gets shot down during air combat. After each trial, the game was reset.

\subsection{Design and Procedure}

Our study design was based, in part, on the study by Kulshreshth et al.[5]. We chose a between subjects design to avoid any effects of learning on user performance, where the independent variable was head tracking (with or without) and the dependent variables were the various scoring metrics used in each game. We wanted some additional information about the use of head tracking in video games for those who played the games without head tracking. Thus, we chose to have those participants who played without head tracking, pick one game to try with head tracking in order to gather their reactions. Both the quantitative and qualitative data was explored collectively as well as according to the two player expertise groupings (casuals and experts).

\subsubsection{Quantitative and Qualitative Metrics}

For each game, we tracked quantitative data that we felt was a good indication of how well users performed. Quantitative metrics are summarized in Table 1. 
Table 2 Post-game Questionnaire. Participants answered these questions on a 7 point Likert scale after playing each game. We used this data for qualitative analysis.

\begin{tabular}{|l|l|}
\hline \multicolumn{2}{|c|}{ Postgame Questions } \\
\hline Q1 & To what extent did the game hold your attention? \\
\hline Q2 & How much effort did you put into playing the game? \\
\hline Q3 & Did you feel you were trying your best? \\
\hline Q4 & To what extent did you lose track of time? \\
\hline Q5 & Did you feel the urge to see what was happening around you? \\
\hline Q6 & $\begin{array}{l}\text { To what extent you enjoyed playing the game, rather than some- } \\
\text { thing you were just doing? }\end{array}$ \\
\hline Q7 & To what extent did you find the game challenging? \\
\hline Q8 & How well do you think you performed in the game? \\
\hline Q9 & To what extent did you feel emotionally attached to the game? \\
\hline Q10 & To what extent did you enjoy the graphics and the imagery? \\
\hline Q11 & How much would you say you enjoyed playing the game? \\
\hline Q12 & Would you like to play the game again? \\
\hline
\end{tabular}

Table 3 Head Tracking Questionnaire. Participants responded to statements 1-4 on a 7 point Likert scale. Questions 5-10 were multiple choice and open ended questions to gauge the users perception of the effects of head tracking.

\begin{tabular}{|c|l|}
\hline \multicolumn{2}{|c|}{ Head Tracking Questions } \\
\hline Q1 & Head Tracking improved the overall experience of the game. \\
\hline Q2 & I would choose to play head tracked games over normal games. \\
\hline Q3 & I felt that head tracking enhanced the sense of engagement I felt. \\
\hline Q4 & Head Tracking is a necessity for my future game experiences. \\
\hline Q5 & Did head tracking help you perform better in the games? \\
\hline Q6 & Which games did it help you in? \\
\hline Q7 & How did it help you in those games? \\
\hline Q8 & Did head tracking decrease your performance in the games? \\
\hline Q9 & Which games did it decrease your performance in? \\
\hline Q10 & How did it decrease your performance in those games? \\
\hline
\end{tabular}

In Arma II, survival time and number of enemies shot were tracked as performance metrics. In Dirt 2, we recorded race completion time and rank in the race. In Microsoft Flight, we recorded the game score. The player was scored on the basis of how many balloons it passed through, if proper speed was maintained while landing, and if the plane landed on runway. In case of a plane crash, this game does not show the final score, but does show the points the player gets for each task while playing. We used this to calculate the final score. In Wings of Prey, number of enemies shot, time taken and game score were tracked as performance metrics.

For the qualitative data, all participants filled out an immersion questionnaire [4] (see Table 2) upon completion of all trials of each game. Responses were measured on a 7 point Likert scale $(1=$ most negative response, $7=$ most positive response). Upon completion of all experimental tasks, participants were given a survey to determine how head tracking affected their gaming experience (see Table 3), whether they preferred to play the games with head tracking, and if head tracking helped or hurt their performance.

\subsubsection{Procedure}

The experiment began with the participant seated in front of the TV and the moderator seated to the side. Partic-
Table 4 Two-way ANOVA analysis for Arma II. Significant differences based on head tracking mode.

\begin{tabular}{|c|c|c|}
\hline Source & Enemies Shot & Time \\
\hline $\mathrm{HTM}$ & $F_{1,36}=4.205, p<0.05$ & $F_{1,36}=5.764, p<0.05$ \\
\hline $\mathrm{EXP}$ & $F_{1,36}=3.577, p=0.067$ & $F_{1,36}=3.812, p=0.59$ \\
\hline $\mathrm{HTM} \times \mathrm{EXP}$ & $F_{1,36}=0.3611, p=0.440$ & $F_{1,36}=4.656, p<0.05$ \\
\hline
\end{tabular}

ipants were given a standard consent form that explained the study. They were then given a pre-questionnaire that focused on their gaming expertise. Participants were then presented with the games in random order (Latin Squares design). Half the participants played the games without head tracking (control group) and half played with head tracking (experimental group). The moderator would present the game and give instructions to the participant as to what they needed to accomplish in the game and what their goals were. They were also instructed on how to use the Xbox 360 controller. During the experiment, the moderator recorded quantitative data using scores from the games and a stopwatch for timing information (if not already provided by the game). After each game, the participant filled out a postquestionnaire with questions about their experiences with the game. If the participants played the four games in the non-head-tracked condition, they then selected one game to play with head tracking. All participants were given a final post-questionnaire about their experiences with head tracking.

\section{RESULTS AND ANALYSIS}

We broke up the participants in each group (head tracked and non-head tracked group) into casual gamers (11 participants in the head tracked group, 11 participants in the nonhead tracked group) and expert gamers (9 participants in the head tracked, 9 participants in the non-head tracked group). To analyze the performance data, a two-way ANOVA was conducted that examined the effect of game-play expertise (EXP), casual or expert, and the head tracking mode (HTM), present $(\mathrm{H})$ or absent $(\mathrm{NH})$, on the average (of the three trials) user performance (see Table 1 for metrics used for each game). We did a post-hoc analysis using independent sample t-tests. We used Holm's sequential Bonferroni adjustment to correct for type I errors [3] and the Shapiro-Wilk test to make sure our data is parametric. We also wanted to see whether there was learning taking place in the form of game play improvement. We looked at the improvement in the performance measures for each game from the first user run to their last run using a repeated measures ANOVA. Finally we wanted to look at the participant's perception of their performance through the post questionnaires. To analyze this Likert scale data, we used the Mann-Whitney test. For all of our statistical measures, we used $\alpha=0.05$. In all graphs error bars represents $95 \%$ confidence interval.

\subsection{Arma II}

Table 4 shows the results of a two-way ANOVA analysis for Arma II. Although this table shows some significance based on head tracking mode (HTM), the post-hoc analysis results were not significant. Experts in the head tracking group $(\mathrm{H})$ survived significantly $\left(t_{16}=31.94, p<0.01\right)$ longer than the experts in the non-head tracking group (NH) (see Figure 2). For score improvements, neither casual gamers nor expert 


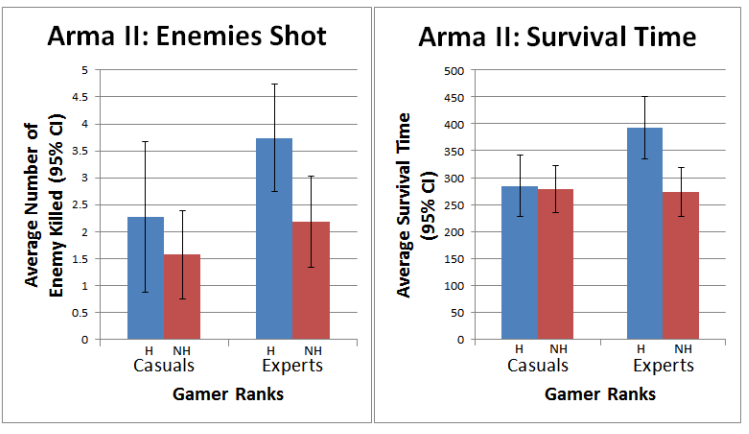

Figure 2 Arma II: Differences in the average number of enemy shot and survival time between the two head tracking modes ( $\mathrm{H}$ : head tracked, $\mathrm{NH}$ : Non-head tracked) in the two gamer categories. Expert gamers performed significantly better with head tracking in terms of survival time.

Table 5 Two-way ANOVA analysis for Dirt 2. Significant differences in rank based on gaming expertise was found.

\begin{tabular}{|c|c|c|}
\hline Source & Race Time & Rank \\
\hline $\mathrm{HTM}$ & $F_{1,36}=0.001, p=0.980$ & $F_{1,36}=0.003, p=0.953$ \\
\hline $\mathrm{EXP}$ & $F_{1,36}=3.738, p=0.061$ & $F_{1,36}=7.467, p<0.01$ \\
\hline $\mathrm{HTM} \times \mathrm{EXP}$ & $F_{1,36}=0.090, p=0.765$ & $F_{1,36}=0.346, p=0.560$ \\
\hline
\end{tabular}

gamers showed any significant improvements, from the first trial to the last trial, in terms of number of enemies shot and survival times. For the questionnaire data, people thought that the game was too challenging $(\bar{x}=6.5, \sigma=0.88)$ and they performed badly $(\bar{x}=2.4, \sigma=1.28)$ in the game. When broken down based on gamer ranks, no significant differences were found on any question in the qualitative data between the two head tracking groups.

\subsection{Dirt 2}

A two-way ANOVA analysis shows (see Table 5) significance in the rank based on game expertise. Gamers in the expert group $(\bar{x}=2.75, \sigma=1.77)$ scored significantly $\left(t_{38}=2.794, p<0.01\right)$ better ranks in the race (lower is bet-

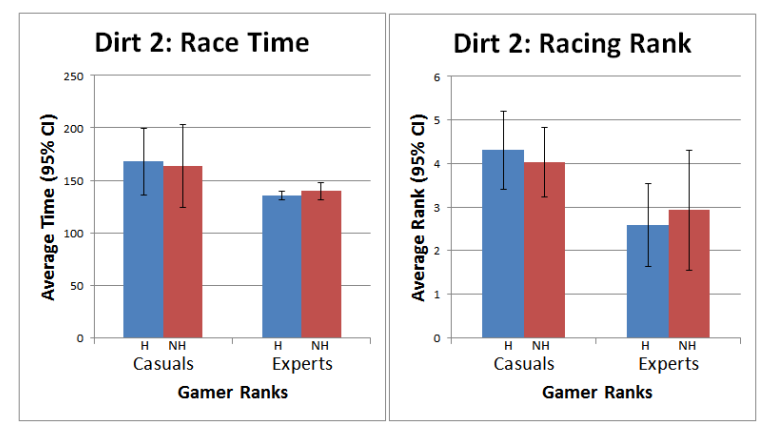

Figure 3 Dirt2: Differences in the average race time and average rank (lower is better) between the two head tracking modes (H: head tracked, NH: Nonhead tracked) in the two gamer categories. Expert gamers took less time and scored better rank with head tracking.
Table 6 Two-way ANOVA analysis for Microsoft Flight. No Significance was found.

\begin{tabular}{|c|c|}
\hline Source & Game Score \\
\hline $\mathrm{HTM}$ & $F_{1,36}=0.021, p=0.886$ \\
\hline $\mathrm{EXP}$ & $F_{1,36}=2.276, p=0.140$ \\
\hline $\mathrm{HTM} \times \mathrm{EXP}$ & $F_{1,36}=0.717, p=0.403$ \\
\hline
\end{tabular}

ter) than the casual gamers $(\bar{x}=4.16, \sigma=1.42)$. For score improvements, casuals in the head tracking group significantly improved their racing time $\left(F_{2,9}=5.354, p<0.05\right)$, from 188.72 seconds $(\sigma=81.14)$ in the first trial to 152.72 seconds $(\sigma=33.72)$ in the third trial, and $\operatorname{rank}\left(F_{2,9}=\right.$ $71.40, p<0.05)$, from $5.36(\sigma=1.50)$ in the first trial to $3.81(\sigma=1.83)$ in last trial. Casuals in the non-head tracking group significantly improved their racing time as well $\left(F_{2,9}=8.449, p<0.05\right)$, from 171.36 seconds $(\sigma=73.87)$ in the first trial to 157.36 seconds $(\sigma=63.75)$ in the third trial, and rank $\left(F_{2,9}=4.244, p<0.05\right)$, from $5.00(\sigma=1.41)$ in the first trial to $3.09(\sigma=2.07)$ in last trial. This translates to $19.07 \%$ improvement for head tracking group compared to $8.16 \%$ for non-head tracking group in terms of time, and $28.91 \%$ improvement for head tracking group compared to $38.20 \%$ for non-head tracking group in terms of game rank. Experts in the head tracking group did not show any significance improvements in racing time or rank. Experts in the non-head tracking group significantly improved their racing time $\left(F_{2,7}=5.048, p<0.025\right)$, from 146.55 seconds $(\sigma=19.04)$ in the first trial to 133.22 seconds $(\sigma=8.58)$ in the third trial, but no significance was found for rank improvement.

For the qualitative data, Dirt 2 held significantly more $(Z=$ $-2.028, p<0.05)$ attention for the head tracking group $(\bar{x}=6.45, \sigma=0.759)$ compared to the non-head tracking group $(\bar{x}=5.7, \sigma=1.380)$. All the participants thought they were trying their best $(\bar{x}=6.10, \sigma=1.277)$ to play the game. Casuals in the head tracking group thought that they put in significantly more effort $(Z=-1.96, p<0.05)$ to play this game, were significantly less $(Z=-1.997, p<0.05)$ distracted, and were trying their best $(Z=-2.144, p<0.05)$, compared to the non-head tracked group. Significantly more people $(Z=-1.97, p<0.05)$ in the casual head tracking group than in the casual non-head tracked group thought that they would like to play the game again. In the case of expert gamers, the head tracking group enjoyed the graphics and imagery significantly more $(Z=-2.012, p<0.05)$ than the non-head tracked group.

\subsection{Microsoft Flight}

No statistically significant differences were found based on head tracking mode or the gamer ranks (see Table 6). Casuals in the head tracking group did not show any significant score improvements, but the casuals in the non-head tracked group significantly improved $\left(F_{2,9}=4.865, p<0.05\right)$, their score from $859.09(\sigma=396.11)$ in the first trial to 995.45 $(\sigma=332.00)$ in their last trial. In case of experts, the head tracked group significantly improved $\left(F_{2,9}=3.811, p<\right.$ $0.05)$, their score from $966.66(\sigma=271.569)$ in the first trial to the maximum possible score of $1150.0(\sigma=0)$ in their last trial, while the non-head tracked group significantly improved $\left(F_{2,9}=8.413, p<0.01\right)$, their score from 761.11 $(\sigma=356.87)$ in the first trial to $1122.22(\sigma=66.66)$ in their 


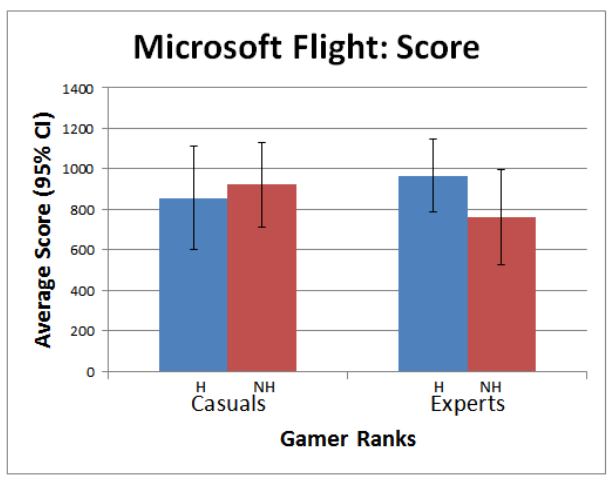

Figure 4 Microsoft Flight: Differences in the game score between the two head tracking modes $(\mathrm{H}$ : head tracked, NH: Non-head tracked) in the two gamer categories. Casual gamers performed slightly better without head tracking but expert gamers performed slightly better with head tracking.

Table 7 Two-way ANOVA analysis for Wings of Prey. Difference in time due to head tracking mode and number of enemies shot due to gaming expertise was found.

\begin{tabular}{|c|c|c|}
\hline Source & Enemies Shot & Time \\
\hline $\mathrm{HTM}$ & $F_{1,36}=0.077, p=0.783$ & $F_{1,36}=5.014, p<0.05$ \\
\hline $\mathrm{EXP}$ & $F_{1,36}=6.271, p<0.05$ & $F_{1,36}=2.093, p=0.157$ \\
\hline $\mathrm{HTM} \times \mathrm{EXP}$ & $F_{1,36}=2.080, p=0.158$ & $F_{1,36}=1.325, p=0.257$ \\
\hline
\end{tabular}

last trial. This translates to $18.97 \%$ improvement for head tracking group compared to $47.44 \%$ for non-head tracking group.

For the qualitative data, the game held the attention of all the participants $(\bar{x}=5.925, \sigma=1.047)$ and all participants thought that they tried their best $(\bar{x}=5.975, \sigma=1.329)$. The head tracked group enjoyed the game significantly more $(Z=-2.564, p<0.05)$ and thought that they performed significantly well $(Z=-2.689, p<0.05)$, when compared to non-head tracked group. When broken down based on gamer ranks, no significant differences were found between the two head tracking groups for casual gamers. But, for expert gamers, head tracked group enjoyed the game significantly more $(Z=-2.473, p<0.05)$ than the non-head tracked group.

\subsection{Wings of Prey}

A two-way ANOVA analysis of the Wings of Prey is shown in Table 7 . The head tracked group $(\bar{x}=245.56, \sigma=34.79)$ took slightly less $\left(t_{38}=-2.096, p=0.043\right)$ time compared to the non-head tracked group $(\bar{x}=266.45, \sigma=27.82)$ but the results were not significant due to the post-hoc correction. However, experts in the head tracked group $(\bar{x}=231.51, \sigma=$ $34.97)$ took significantly less $\left(t_{16}=-2.301, p<0.05\right)$ time compared to the experts in the non-head tracked group $(\bar{x}=$ $264.85, \sigma=25.80$ ) (see Figure 5). Experts $(\bar{x}=4.12, \sigma=$ $2.36)$ shot significantly more $\left(t_{38}=-2.501, p<0.025\right)$ enemy planes than casual gamers $(\bar{x}=5.68, \sigma=1.31)$. For score improvement, no significant differences in terms of enemies shot or time taken were found for either casual gamers or expert gamers.

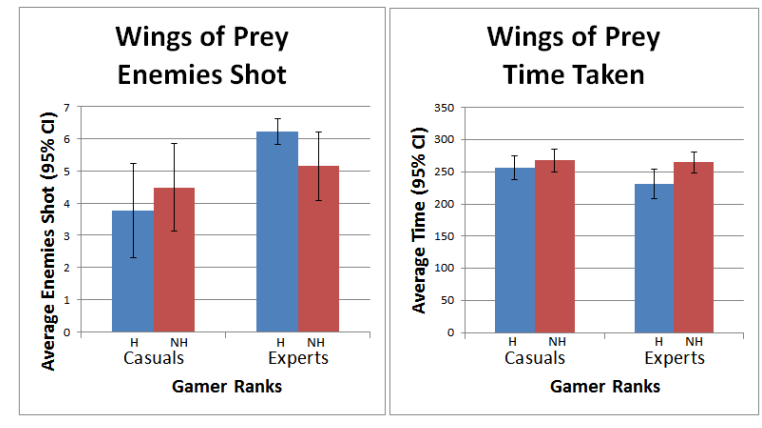

Figure 5 Wings of Prey: Differences in the average number of enemies shot and time taken between the two head tracking modes (H: head tracked, $\mathrm{NH}$ : Non-head tracked) in the two gamer categories. Expert gamers shot slightly more enemies and took significantly less time with head tracking.

For qualitative data, the game held the attention of all the participants $(\bar{x}=6.05, \sigma=1.153)$ and all participants thought that they tried their best $(\bar{x}=6.15, \sigma=1.291)$. Qualitatively, no significant differences were found between the head tracked and non head tracked groups. When broken down based on gamer ranks, there were also no significant differences.

\subsection{Head Tracking Questions}

Out of the 20 participants in the non-head tracked group, three chose to play Arma II, five chose to play Dirt2, three chose to play Microsoft Flight, and nine chose to play Wings of Prey. All three participants who played Arma II thought that head tracking helped them. Only one out of five participants who played Dirt 2 thought that it helped them. Two participants out of three who played Microsoft Flight thought that it helped them. Finally, six out of nine participants who played Wings of Prey thought that it helped them.

Out of the 20 participants from the head tracked group that played all games with head tracking, 19 participants thought that it gave them an advantage in at least one of the games and 13 thought that it hurt their performance in at least one of the games. Eight in Arma II, seven in Dirt 2, and only one in Wings of Prey thought that head tracking hurt their performance. No one thought that head tracking hurt their performance in Microsoft Flight.

All the participants filled out a questionnaire about their experience with head tracking (see Table 3), responding to questions Q1-Q4 on a 7 point Likert scale (1=Strongly Disagree, $7=$ Strongly Agree). All the participants agreed that head tracking improved their overall gaming experience $(\bar{x}=5.05, \sigma=1.83)$ and enhanced the sense of engagement they felt $(\bar{x}=5.30, \sigma=1.69)$. However most participants did not think that head tracking was a necessity for their future gaming experience $(\bar{x}=3.32, \sigma=1.93)$. We did not find any statistically significant differences when data was divided across gamer ranks or head tracking modes.

\section{DISCUSSION}

Hypothesis testing results for each game are summarized in Table 8. Hypothesis H1 is true only for expert gamers in case of Arma II and Wings of Prey . Hypothesis H2 was always 
Table 8 Summary of hypothesis (see section 4) testing results for all games in the two gamer ranks. ( $\mathrm{T}=$ True and $\mathrm{F}=$ False)

\begin{tabular}{|c|c|c|c|c|c|c|}
\hline \multirow{2}{*}{ Game } & \multicolumn{3}{|c|}{ Casual Gamers } & \multicolumn{3}{c|}{ Experts Gamers } \\
\cline { 2 - 7 } & H1 & H2 & H3 & H1 & H2 & H3 \\
\hline Arma II & F & F & F & T & F & F \\
\hline Dirt 2 & F & F & T & F & F & F \\
\hline Microsoft Flight & F & F & F & F & F & T \\
\hline Wings of Prey & F & F & F & T & F & F \\
\hline
\end{tabular}

found to be false which means that head tracking did not help in learning the games faster. Hypothesis H3 was true for casual gamers in Dirt 2 and expert gamers in Microsoft Flight. We noticed large variability, as indicated by large error bars in charts, in our user performance data which could be due to few factors. One factor may be different gaming abilities of the users, an expert FPS gamer may not necessarily be an expert in flight simulation or racing games. Another factor could be insufficient game training time before the experiment.

Based on our quantitative data, we can see that head tracking provided significant performance advantages only for expert gamers for Arma II (better survival time) and Wings of Prey (better time and more number of enemies shot). No other significant advantages were found in the other games we tested. Both Arma II \& Wings of Prey are shooting games and in both games head tracking is useful to find enemies around the player's current position. In Arma II, gamers found it useful and natural to rotate their head to look around and move closer to the screen to zoom-in and iron-sight. In the case of Dirt 2, the user had to look forward most of the time and rotating one's head makes it difficult to focus on the road, especially at fast speeds. So, head tracking turned out to be not that useful for this game. In the case of Microsoft Flight, although the head tracking added depth perception and a sense of realism to the game, the game itself was slow paced and not difficult to play. So, users did equally well and it did not matter much if head tracking was present or not.

While examining learning effects (e.g., score improvement with each game trial), we noticed that there were significant improvements in some cases when the two groups (head tracked vs non-head tracked) were analyzed separately. However, head tracking usage did not enhance learning, when compared to non-head tracked environment, and in some cases negatively affected learning (e.g., experts in Dirt 2 learned faster without head tracking). But, experts in the head tracking group for Dirt 2 already started with a high score and did not improve much. In the case of Microsoft Flight, the casual non-head tracked group and both expert groups (head tracked vs non-head tracked) improved their score significantly. For Arma II and Wings of Prey, we did not notice any significant improvements across runs. In the case of Arma II, the head tracked group already started with a higher score than the non-head tracked group and did not improve significantly with trials. In the case of Wings of Prey, casual gamers in the head tracked group started with a lower score than the non-head tracked group and both groups did not improve much with repeated attempts. However, expert gamers had a higher score in the head tracked group than the non-head tracked group but it did not improve much with repeated attempts.
Another important factor that could affect our results is the fact that head tracking was an added feature in all the games we tested. So it was up to the user whether to take advantage of head tracking or not. While expert gamers could make better use of head tracking, casual gamers appeared to focus more on games basics and did not pay much attention to head tracking. This may explain why casual gamers performed almost equally well in both the groups (head tracked vs non-head tracked). So far head tracking devices are not as successful as motion controllers (e.g., Sony Move or Nintendo Wii). Games which make use of motion controllers usually provide in-game usage instruction (e.g. a tutorial when the game starts or hints while playing) for their effective use but we found this missing in case of head tracked games we tested. Some instructions could have helped users make better use of head tracking while playing.

Based on our qualitative data, in some games we found significant differences in the two user groups (head tracked vs non-head tracked). Head tracking was perceived to be significantly more enjoyable in Microsoft Flight. Casual users had to put significantly more efforts to play Dirt 2 with head tracking. We did not find any significant differences in Arma II and Wings of Prey. In general, almost all participants were not familiar with the games we tested, and the users played for a short period of time (60 to $80 \mathrm{~min}-$ utes). This may explain why we did not notice significant differences in qualitative data for most games.

Additionally, our qualitative data indicates that head tracking is perceived to be more enjoyable for slow paced games and could harm user performance when used in fast paced games. Our results contradict previous findings [7, 11, 15], which indicate that although intuitive and enjoyable, head tracking does not provide significant performance benefits. The main reason for these differences could be the choice of game tasks we assigned to participants or the head tracking system used for this study. All the games we tested had native head tracking support and currently there is a limited selection of game genres (Racing, Flight Simulator, and First Person Shooter) that support head tracking, so we need to explore more head tracked based interaction techniques to be able to use them in more game genres. This could be achieved by including tasks in the games which can only be achieved by head tracked-based interaction and bonus points could be given for these tasks. This would force users to use head tracking and help them learn new head tracking based interaction techniques. This could be useful, especially, in the initial phases until head tracking becomes a very commonly used gaming accessory. Based on our findings and observations, we have the following recommendations to game designers:

- Make use of head tracking in FPS and air-combat games because these games have tasks that could benefit from head tracking usage.

- Include instructions/hints while playing games to guide gamers to make optimal use of head tracking. Most people are used to playing games with traditional button based controllers, so most of the time they forget to use head tracking. We think, instructions/hints while playing would remind them of the presence of head tracking.

- Limit head tracking usage in racing games. Head tracking usage could be distracting for racing games. 
Note that our study did have some limitations. Due to the nature of experiment and time limitations, it was difficult to balance (in terms of gaming abilities) the participants across the two groups (head tracked vs non-head tracked). Although we had same number of expert users in the two groups, the casual head tracked group had more beginners than the casual non-head tracked group. This disproportion could have skewed some of our results. In addition, unlike previous work $[10,13,14]$, the games we tested were complex so it may have been difficult for users to use head tracking effectively and learn how to play the games at the same time. This could have had an affect on performance results.

\section{CONCLUSION AND FUTURE WORK}

We have presented a study exploring the effects of head tracking on user performance in head tracking enabled modern video games. We observed that head tracking could provide significant performance advantages for certain games (Arma II and Wings of Prey) depending upon game genres and gaming expertise. Our results indicate that head tracking is useful in shooting games (FPS, air combat etc.) and it is not a good idea to use it in a fast paced racing games. However, not all users benefit equally well with head tracking. Casual gamers do not benefit significantly from head tracking, but expert gamers can perform significantly better when head tracking is present. A possible reason is that casual gamers focus more on the basic games mechanics and do not pay much attention to a more advanced feature like head tracking. Our qualitative results indicate that head tracking is more enjoyable for slow paced video games (e.g. flight simulation games) and it might hurt performance in fast paced modern video games (e.g. racing games).

Our study is a preliminary step towards exploring the effectiveness of head tracking in realistic game scenarios. Clearly, further research with more game genres and head tracking techniques is required to further validate our results. In the future, we will continue to explore how head tracking could be made more enjoyable and effective in modern video games including how to better enhance casual gamer's performance. We also plan to explore whether head tracking is a better depth cue compared to 3D stereo and the effectiveness of simultaneously using head tracking and 3D stereo in modern video games.

\section{ACKNOWLEDGMENTS}

This work is supported in part by NSF CAREER award IIS0845921 and NSF awards IIS-0856045 and CCF-1012056. We would also like to thank the members of ISUE lab for their support and the anonymous reviewers for their useful comments and feedback.

\section{REFERENCES}

[1] M. Ashdown, K. Oka, and Y. Sato. Combining head tracking and mouse input for a gui on multiple monitors. In CHI '05 Extended Abstracts on Human Factors in Computing Systems, CHI EA '05, pages 1188-1191, New York, NY, USA, 2005. ACM.

[2] M. Bajura, H. Fuchs, and R. Ohbuchi. Merging virtual objects with the real world: seeing ultrasound imagery within the patient. SIGGRAPH Comput. Graph., 26(2):203-210, July 1992.
[3] S. Holm. A simple sequentially rejective multiple test procedure. Scandinavian Journal of Statistics, 6(2):65-70, 1979.

[4] C. Jennett, A. L. Cox, P. Cairns, S. Dhoparee, A. Epps, T. Tijs, and A. Walton. Measuring and defining the experience of immersion in games. International Journal of Human-Computer Studies, 66,9:641-661, 2008.

[5] A. Kulshreshth, J. Schild, and J. J. LaViola Jr. Evaluating user performance in $3 \mathrm{~d}$ stereo and motion enabled video games. In Proceedings of the International Conference on the Foundations of Digital Games, pages 33-40, New York, NY, 2012. ACM.

[6] J. Lee. Hacking the nintendo wii remote. J. IEEE Pervasive Computing, 7(3):39-45, 2008.

[7] S. Marks, J. Windsor, and B. Wunsche. Evaluation of the effectiveness of head tracking for view and avatar control in virtual environments. In Image and Vision Computing New Zealand (IVCNZ), 2010 25th International Conference of, pages 1-8, Nov 2010.

[8] NaturalPoint. Trackir 5. http://www naturalpoint. com/trackir/products/trackir5/.

[9] J. Rekimoto. A vision-based head tracker for fish tank virtual reality-vr without head gear. In Virtual Reality Annual International Symposium, 1995. Proceedings., pages 94 -100, mar 1995.

[10] T. Sko and H. Gardner. Head tracking in first-person games: Interaction using a web-camera. In Human-Computer Interaction - INTERACT 2009, volume 5726 of Lecture Notes in Computer Science, pages 342-355. Springer Berlin / Heidelberg, 2009.

[11] R. J. Teather and W. Stuerzlinger. Exaggerated head motions for game viewpoint control. In Proceedings of the 2008 Conference on Future Play: Research, Play, Share, Future Play '08, pages 240-243, New York, NY, USA, 2008. ACM.

[12] M. Terlecki and N. Newcombe. How important is the digital divide? the relation of computer and videogame usage gender differences in mental rotation ability. Sex Roles, 53:433-441, 2005.

[13] S. Wang, X. Xiong, Y. Xu, C. Wang, W. Zhang, $\mathrm{X}$. Dai, and D. Zhang. Face-tracking as an augmented input in video games: enhancing presence, role-playing and control. In Proceedings of the SIGCHI Conference on Human Factors in Computing Systems, CHI '06, pages 1097-1106, New York, NY, USA, 2006. ACM.

[14] J. Yim, E. Qiu, and T. C. N. Graham. Experience in the design and development of a game based on head-tracking input. In Proceedings of the 2008 Conference on Future Play: Research, Play, Share, Future Play '08, pages 236-239, New York, NY, USA, 2008. ACM.

[15] D. Zhu, T. Gedeon, and K. Taylor. Keyboard before head tracking depresses user success in remote camera control. In Human-Computer Interaction, INTERACT 2009, volume 5727 of Lecture Notes in Computer Science, pages 319-331. Springer Berlin / Heidelberg, 2009. 